\title{
Epidemiology and outcomes from out-of-hospital cardiac arrest in the province of Lecce: a
} report of a 5-year experience.

Gaetano Tammaro ${ }^{1}$, Enzo Picconi ${ }^{2}$, Maurizio Scardia ${ }^{1}$, Stefano Scardia ${ }^{3}$, Cesare Sabetta ${ }^{1}$, Daniele Antonaci ${ }^{1}$, Domenica Rita Ruggeri ${ }^{1}$, Luca Tortorolo ${ }^{2}$.

${ }^{1} 118$ Lecce Emergency Services Operations Center, Lecce, Italy.

${ }^{2}$ Department of Intensive Care Medicine and Anesthesiology, Fondazione Policlinico Universitario A. Gemelli Università Cattolica del Sacro Cuore, Rome, Italy.

${ }^{3}$ Department of Internal Medicine, Regional Hospital of Lugano, Lugano, Switzerland.

\section{Introduction}

Out-of-hospital cardiac arrest (OHCA) is one of the leading causes of death and disability worldwide. In 2013, an OHCA registry was set up by the Territorial Emergency Medical Service (EMS) of Lecce, Italy; this public service operates in an area of 814,495 inhabitants, increasing up to about 1,600,000 during the summer. The aim of this study is to assess the Lecce OHCA registry to report the epidemiology and the rates of survival after OHCA, with regard to EMS response times.

Materials and methods

A prospective observational study from Lecce OHCA registry was conducted. The registry contains data about all the OHCA attended by the EMS between 1st January, 2013 and 31st December, 2017. Data were recorded chronologically by ambulance crew and included patient characteristics (age and sex), arrest features, EMS response times, treatment and patient outcomes (return of spontaneous circulation [ROSC] or death).

\section{Results}

4203 OHCA cases of EMS treated cardiac arrests were recorded in the study period (103 per 100,000 of resident population per year). The mean age was 76.1 (SD 16.5 ) years and $44.8 \%$ were female. The initial rhythm at emergency team arrival was mainly asystole (87.2\%); ventricular fibrillation or tachycardia was observed in $9.4 \%$, pulseless electrical activity in $3.4 \%$. ROSC at hospital transfer was evident in $4.5 \%(n=191)$ of cases. Mean time from call to ambulance sending was of 83 seconds (no difference between survivors and dead). Mean time from EMS call to ambulance arrival was of 15 minutes and 48 seconds ( 12 minutes and 2 seconds for survivors and 15 minutes and 58 seconds for dead, $\mathrm{p}=0,04)$.

\section{Conclusion}

In the province of Lecce, OHCA is a major cause of death, with a low survival rate. Information about epidemiology and EMS response times can contribute to improve survival and patients outcome. 